\title{
A Comprehensive Study of Real-Time Vacant Parking Space Detection Towards the need of a Robust Model
}

\author{
Rifath Mahmud, A.F.M Saifuddin Saif, and Dipta Gomes
}

\begin{abstract}
Detection of vacant parking space is becoming a challenging task gradually. Space utilization and management of vehicle space is now a demandable field of research. Searching for an empty parking space in congested traffic is a timeconsuming process. The existing vacant parking space detection methods are not robust or generalized for images captured from different camera viewpoints. Finding a proper parking space in a busy city is really a challenging issue and people are facing this problem on a daily basis. The main purpose of this research is to comprehensively discuss the previous researches of vacant parking space detection and compare them from different aspects. Methods used in previous researches are descriptively discussed along with their advantages and disadvantages. The frameworks of previous researches were compared on six generalized phases and the experimental results are compared in terms of dataset, accuracy, processing time and other performance measures. This research also focuses on the challenges of vision-based vacant parking space detection which will contribute to future researches and researchers can work to overcome these challenges.
\end{abstract}

Index Terms - Object Detection, Neural Networks, Parking space management, Segmentation

\section{INTRODUCTION}

Worldwide, the number of people increasing day by day and the living style of people all over the world is also getting better. One of the big factors of the lifestyle of modern age people is transportation and that's the reason the number of people owning a motor vehicle is also increasing. As a result,

Rifath Mahmud

Lecturer, Department of Computer Science,

American International University Bangladesh (AIUB)

Email: rifath.mahmud@aiub.edu

A.F.M. Saifuddin Saif

Senior Assistant Professor,

American International University Bangladesh (AIUB)

Email: saif@aiub.edu

Dipta Gomes

Lecturer, Department of Computer Science,

American International University Bangladesh (AIUB)

Email: diptagomes@gmail.com the number of vehicles is increasing. Along with this increasing number of vehicles, requirements of parking are becoming a problematic issue globally. So, searching for a parking area that is suitable for the motorist or the owner in a populated metropolitan city is becoming extremely difficult. Severe congestion of traffic can happen for not being able to find proper parking space in the desired area. But if the driver or user is able to get information about this unavailability or availability of parking spaces, he or she will be able to look for parking space somewhere else and would not need to enter parking area that does not have an available parking space.

Parking systems are manually handled by a human individual. For this reason, it is time-consuming and also inaccurate. People also feel frustrated to find parking space. But many of the parking areas may have parking space that nearby drivers are unaware of. So, the automatic parking system is an emerging and attracted field for researchers on computer vision to contribute to this technology [1]. The systems that are previously developed are mainly on the basis of image segmentation or machine learning mainly Support Vector Machines (SVM), Neural Networks (NN) over spot patches. But it is possible to use these algorithms for the proper detection of automatic parking management systems because of the evolution in the last years of object detection algorithms [2].

Providing enough parking for visitors is one of the main issues in many real-life scenarios. Safe and secure parking lots with a enough spaces can increase visitor's loyalty and attract visitors to visit in many places more frequently. This research aims to set milestones that need to be achieved to develop a model for real-time vacant parking space detection for efficient and smart parking.

The main purpose of this research is to find existing methods and frameworks and evaluate them so that in future researches a more robust model can be developed for general purpose use, which can help the motorist in efficiently finding vacant parking space. In addition, a reservation feature can be provided to reserve the space if the vehicle is in a certain range of the parking area. The model should be able to find a total number of parking spots in a parking area and how many of them are occupied and how many of them are unoccupied and this information needs to visually represent for the targeted users in real-time. 


\section{CORE BACKGROUND STUDY}

D. Di Mauro et al. [3] mainly compared the previous works on parking slot detection for both stall-based and stall-free scenarios. Classification methods can be applied when the geometry of the scene is known and image segmentation can be used when the configuration is unknown. A method named temporal smoothing which can improve the outcome of object detection and image segmentation is mentioned. But there is no specific method is proposed for parking spot detection. F. Dornaika et al. [4] proposed a machine learning technique named handcrafted approach parking spot detection. Pyramid multi-level descriptor (PMLD) is used to extract features from different image region and various image-based handcrafted and deep learning features are also reviewed. S. Nurullayev et al. [5] proposed a Dilated Convolutional Neural Network based approach to detect available parking space. H. Bura et al. [6] used distributed cameras, edge computing, data analytics, and advanced deep learning algorithms to detect parking spot. Real-time deep learning inference for detection in edge devices are used. This approach can detect the entering of a car, the location and can also charge parking fee associated with the license plate.

R. Nieto et al. [7] used a multicamera based system to detect vehicles and then mapped it to parking spots. Here, some important features like occlusion, illumination changes, and different climatic conditions are considered. The best part of this research is that a real scenario is used to validate the research. $\mathrm{H}$. $\mathrm{Vu}$ et al. [8] used Deep Convolutional and contrastive network to detect parking spot which is mainly custom trained. To extract contrastive and robust features, a Siamese architecture is used and also convolutional Spatial Transformer Network (STN) is used. The multi-task loss function is also exercised for training the network. M. Lee et al. [9] proposed a stereoscopic computer vision with depth perception approach for counting vehicles using a modified disparity histogram. The adjusted distribution map is applied for detecting vehicles. J. Martinez Garcia et al. [10] approached for a modified convolutional neural network $(\mathrm{CNN})$ for classifying images. A MIMO-FMCW radar is utilized to extract slant-range image of parking scenarios.

N. Bibi et al. [1] used Deep Learning ( mAlexNet, mLetNet uses Convolutional Neural Networks) to classify the parking space. The advantage of this method is, the classification phase needs low computational resources, Scalable, and lower cost and versatility but it requires an additional camera and Raspberry usage rather than using security camera in the parking lot. For segmentation mask was built once manually for two cameras (so manual masking will be required for a different view). G. Sarage et al. [11] utilized a geometric features-based approach which consists of one guideline and several parallel separating lines with a fixed distance. There are two kinds of guidelines: a continuous straight line and Tshaped or L-shaped marking points. The false line is Removed and then guideline is detected using a learning-based method. This method outperforms the single bird's eye view method that by many researchers but this method cannot meet the realtime requirement. C. Jang et al. [12] proposed a convolutional network for semantic segmentation for detecting free parking spots. A vertical grid encoding method is used for the same purpose.

\section{REVIEW BASED ON METHODS}

Neural Network and Deep learning are able to solve problems that other methods are struggling to provide solutions in Image detection and recognition. Neural Networks are designed in such a way that they were able to recognize patterns from the correlation and learn the similarities and dissimilarities of data which is trained on. There are many variants of Neural Network, one of the variants is Convolutional Neural Networks (CNN), which is heavily used in the field of Computer Vision. In vision-based vacant parking space detection, methods that use Neural Network and Deep Learning is a technique where researchers develop a classification model by providing a large number of input data to the Neural Network and training on them [13][14]. This type of approach is known as a learning-based approach where the model learns about the different features of the input image throughout the training phases. These features are detected automatically by analyzing input data, CNN tries to take advantage of the spatially local correlation in images, by stacking the feature maps and only connecting each neuron to a small region of the input volume on every epoch. Epoch is a parameter that defines the number of times the learning algorithm will use the training dataset for training [15][16].

Many datasets are required to train the models, but training is costly in respect to hardware and time. There are some robust datasets for vacant parking space detection like PK-Lot introduced by P.R.L. de Almeida et al. [17], CNRPark that are being used by a number of researchers to develop parking spot detection model [5], [18]. Training a Neural Network for detecting parking space would require a computer with a higher specification and will also require more time than other methods. Other method includes approaches like geometric feature-based analysis to detect if a parking spot is free or occupied [19]. Using Image processing techniques geometrical information's are extracted from the images and based on them one can train a simple machine learning algorithms like SVM (Support Vector Machine), Linear Regression, KNN (knearest neighbors), random forest, etc. [16] [20].

One of the challenges of parking space vacancy detection is using only the images to locate all the parking spaces in the parking lot. Some researchers used a fixed mask to segment all the parking spaces then use detection model to check if space is occupied or not. The terminology is that in a parking area only a vehicle will move into a spot or leave a spot but the spot is never changed. So, using a fixed mask gives an advantage of fast segmentation of all parking spaces but the problem is changing camera position or angle will require to change the mask [13] [18].

Background subtraction method uses a mixture of Gaussians to detect and track vehicles and the creation of a transience map to detect the parking and leaving of vehicles is proposed by C. del Postigo et al. [21]. Edge Based methods are also used to detect parking spaces, the methods use edge 


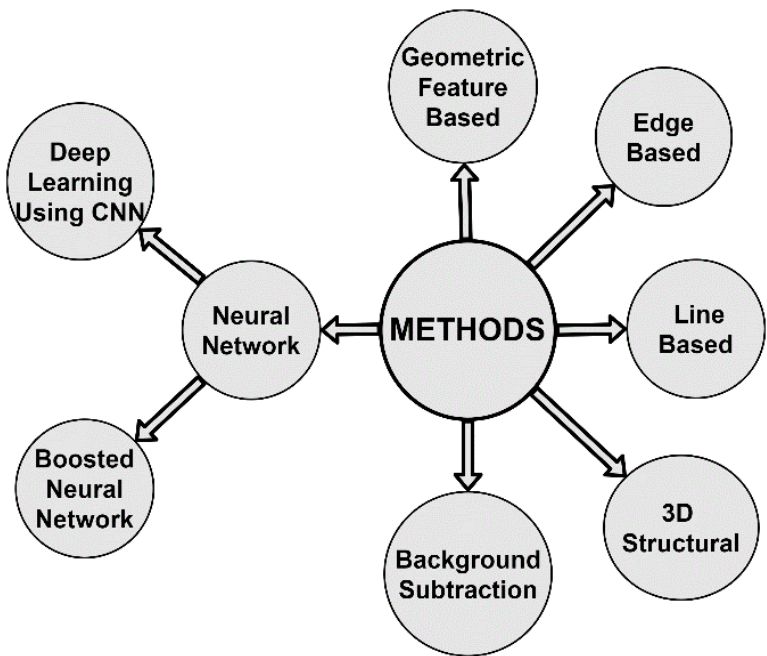

Fig. 1. Existing methods from previous research.

information's to distinguishes between occupied and free space which is decided by the classification algorithm [6]. C. Huang [22] and I. Masmoudi [23] used 3D parking lot structuring. "The method consists of an Image layer, a Patch layer, a Space layer, and a Lot layer. In the Image layer, image patches were selected based on the 3D parking lot structure" and this method can overcome the inter-object occlusion. Around view monitoring system is also used to semantically segment and detect parking spaces in small or indoor parking areas [24].

Here, Error! Reference source not found. illustrates the methods used in previous researches. Issues with these methods are that they do not provide a complete package that is good in terms of automatic segmentation, minimal features extraction for training, lightweight classifiers that require low computation and detection of a parking spot for efficient parking, also a good representation is required for end-users.

TABLE I

EXISTING METHODS WITH ADVANTAGES AND DISADVANTAGES

\begin{tabular}{ccc}
\hline \hline Methods & Advantages & Disadvantages
\end{tabular}

\begin{tabular}{ll}
\hline Neural Network & Faster recognition and \\
[22], [25], [26] & $\begin{array}{l}\text { gives overall accuracy } \\
\text { good. }\end{array}$
\end{tabular}

Convolutional neural network [4], [5], [15], [27]

CNN tries to take
advantage of the spatially
local correlation in images
and also robust and stable.

Geometric Feature-Based [19], [28]

Edge Based [6][29]

This process can recognize the parking slot markings under diverse complex lighting and ground conditions

Mostly does analysis on the edges to extract
Computationally expensive while training and requires powerful machine and time.

Overfitting is a problem of CNNs and change in camera and illumination most CNNs cannot perform well.
Background

Subtraction

[21][28] information.

Line Based

3D Structural

[8][23][22]
Provides well enough results for automatic region extraction where others may rely on Fixed Mask.

Inter-object occlusion and achieve better status inference in many environmental variations and different weather conditions.
Some parking space may not get detected because of light and shadows and also heavily rely on the line drawn in the parking area. $\rightarrow 103 \mathrm{~A} / \mathrm{m}$

A 3D coordinate system is used to structure the parking lot and manually measured location, size, and the shape of each parking space for 3D structuring. So, the problem is if the camera is displaced even if a little bit it creates a huge difference.

Background images should be learned for various lighting conditions which perform well in outdoor condition. with other methods.

\section{REVIEW BASED ON FRAMEWORKS}

This section is divided into six subsections in Figure 1 and comprehensively discussed every section by comparing frameworks of researches done previously.

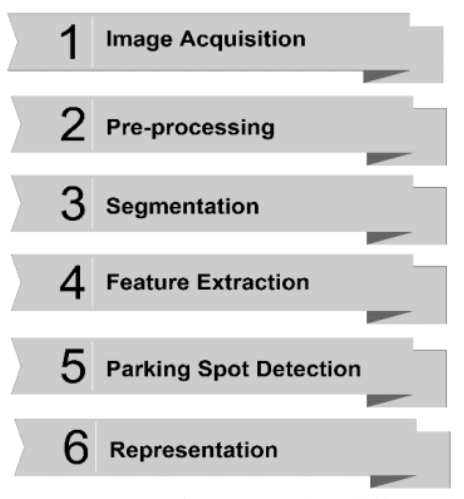

Figure 1: Generalized Framework for Comparing different steps of existing frameworks.

\section{A. Image Acquisition}

There are some popular datasets that were made dedicatedly for parking vacancy detection two of the most popular ones are PKLot Dataset [17] and CNRPark Dataset[18][13]. PKLot consists of 695,900 images and XML file consisting pixel information of occupied or vacant and CNRPark is composed of 12,584 labeled images for training. These images are collected in different weather conditions such as sunny, rainy and cloudy. These datasets are rich in terms of research based on the scenario presented in this paper. The dataset contains images of different weather conditions from various locations of parking places. The parking spaces in the images were annotated over 100 places. Tongji Parking-slot Dataset 2.0 is 
also a good dataset with 5100 images where parking regions enclosing (potential) marking-points are marked [27]. Besides there are a lot of ways to acquire image data for parking space detection. R. Nieto [2] recorded "parking Lot dataset" (PLds) dataset as there was a lack of public parking lot datasets. They collected image in different illumination (day, night, sunrise with shadows) and weather (sunny, rainy) conditions. The dataset consists of two main image sets, a training set used to generate the detector models, and a test set used for the experimental evaluation. There are a lot of researchers who collected data similarly in [22][26][28] based on similar scenarios relating to Car Parking Space, that includes the method of collecting data, Camera Calibration, etc.

\section{B. Pre-processing}

Some of the common pre-processing are normalize the image, noise removing using filtering median filter is mostly used as it is an edge-preserving filtering technique, Grayscale Conversion, Grayscale to Binary image, morphological transformation [11], [31]. Pre-processing is important as there are a number of operations that will be done for segmentation and detection. Images with noise and different illumination variation may cause a huge deal which is reduced through preprocessing.

\section{Segmentation}

Several Segmentation techniques are used in various researches. Finding the boundary lines of parking spaces using line detection algorithms like Hough transformation. In paper [28], Hough transform has been used as well to detect space lines, in which a skillful cell accumulation scheme is used to generate equal-width curves, yielding a more robust and accurate detection result of parking space boundary lines. But in most of the researches, they used a fixed mask which is generated once for each calibrated camera and used to segment out parking spaces [2], [13], [21]. The benefit of using a mask is that it gives accurate segmentation and is really faster than finding all parking space based on line detection. So, the overall process becomes really fast.

\section{Feature Extraction}

Features are extracted from segmented images, these features information are used to find differences between vacant parking space and occupied parking spaces like calculating angle and length of each line in parking spot, etc. [30]. Geometric features like shape are also used by X. Ling et al. [19], [32]. Robust Features are collected because it provides good generalized features which are then used to train the desired model for detection [19], [25].

\section{E. Parking Spot Detection}

Using the extracted features machine learning model are trained for classification of the parking space. But methods that use Deep Learning extracts features automatically and uses them to train itself [15], [18]. Using Deep Learning complex features and correlation can be found that are not found by the human eye. But over fitting is an issue in deep learning that needs to be kept in account [6], [14]. Methods that uses Neural networks are not resource friendly while training the model [15], [26] but they have a good accuracy requires less resources while testing. Also, KNN, SVM, Logistic Regression, Random Forest can be used to train on the collected features from the images [16][20]. These lightweight machine learning algorithms work well on RealTime parking space detection.

\section{F. Representation}

Now the detected parking spaces need to visualize in such a way that the target people understand easily what information is shown. In most of the cases, the parking space detected information is shown by drawing rectangle shapes on the detected parking spots that are not used and that are in use. Free spots can be marked as GREEN as available and occupied spots can be marked as RED used by F. Dornaika et al. [4], [16]. Sometimes instead of providing the real image an abstract view image is created that are represented as colored blocks [22], [33].

\section{REVIEW BASED ON EXPERIMENTAL RESULTS}

In this study a comprehensive review is done on previous researches validations in terms with overall accuracy levels, is good enough for real-time processing, training and testing time in regards of different datasets used by the researchers.

\section{A. Review based on Accuracy Vs Methods}

In most of the researches, authors tend to use Convolutional Neural Networks since the advantage of using CNN is that it gives higher accuracy [13], [14], [34].Some problems relating to $\mathrm{CNN}$ are that it tends to overfit data. On the otherhand, training each pair of parking space and day is randomly chosen as belonging to either the training or evaluation set with equal probability. This prevents multiple images from the same parking space during the same day. Neural network would require powerful hardware. J. Martínez García et al. [10] used different feature extraction method to extract robust features and train classifiers. G. Amato et al. in paper [13] used CNN combining Caffe Deep Learning framework with gradient descend an iterative optimization algorithm with momentum (where, Momentum = 0.9). They used Raspberry Pi 2 Model B, equipped with the standard Raspberry Pi camera module 2 and archives an accuracy of $82.9 \%$ on CNRPark, and 90.4 on PKLot dataset with a learning rate of 0.001. Qian Li et al. in paper [19] utilized the cascade classifier, Haar feature and LBP feature where the AdaBoost method trains the same classifier (weak classifier) for different training sets and then combines these classifiers obtained on different training sets to form a stronger final classifier (strong classifier) and Majority Vote classifier gives a precision of $98.2 \%$ and a recall of $96.8 \%$. In order to test the robustness of the system T. Jensen et al. trained on a single parking area and then tested on both the same parking area image and on parking area images that have not been seen by the model [14]. They mentioned that the lowest accuracy achieved is $95.45 \%$ when they trained on the UFPR05 and tested on 
UFPR04 with Learning Rate $=0.0001$. P.R.L. de Almeida et al. [17] used Support Vector Machine (SVM) classifier and linear scaling normalization. The accuracy of the single classifier dropped from $99.55 \%$ to $84.25 \%$ in the worst case, while a minor loss was observed for the ensemble-based solutions, from $99.64 \%$ to $88.33 \%$ in the worst scenario but they have reached outstanding recognition rates which is around 99\%. M. Ahrnbom et al. [25] divided the PKLot dataset into two sections, one of them is used for training classifiers and other is used for evaluating the classifiers performance. Here each pair of parking space and day is randomly chosen from the dataset so that the data belongs to either the training or evaluation set with equal probability. This prevents multiple images from the same parking space during the same day." They trained two classifiers and they have evaluated and compared them to each other. One of them is Support Vector Machine and another is Logistic Regression where Support Vector Machine achieved an accuracy of 0.9768 and Logistic Regression achieved an accuracy of 0.9868. In Table 2 Experimental results based on Accuracy of different method used in different papers for various datasets are given.

TABLE 2

EXPERIMENTAL RESULTS OF DIFFERENT METHODS ON VARIOUS DATASETS

\begin{tabular}{ll}
\hline \hline \multicolumn{1}{c}{ Methods } & \multicolumn{1}{c}{ Datasets } \\
\hline $\begin{array}{l}\text { Convolutional } \\
\text { neural network }+\end{array}$ & $\begin{array}{l}\text { PKLot Dataset, CNRPark } \\
\text { Deep Learning- } \\
\text { based [13] }\end{array}$ \\
& $\begin{array}{l}\text { consists of 695,900 images } \\
\text { and xml file consisting } \\
\text { pixel information of } \\
\text { occupied or vacant } \\
\text { CNRPark is composed of } \\
\text { 12,584 labeled images for } \\
\text { training }\end{array}$ \\
\end{tabular}

Convolutional

PKLot Dataset

neural network-

based [14]

Convolutional neural networkbased [10]

\begin{tabular}{|c|c|}
\hline $\begin{array}{l}\text { Convolutional } \\
\text { neural network } \\
\text { based CarNet[5] }\end{array}$ & $\begin{array}{l}\text { PKLot Dataset } \\
\text { CNRPark + EXT Dataset }\end{array}$ \\
\hline $\begin{array}{l}\text { Geometric } \\
\text { features based } \\
{[19]}\end{array}$ & $\begin{array}{l}5100 \text { images in test dataset } \\
\text { were captured by a fisheye } \\
\text { camera, and their } \\
\text { resolution was } 600 \times 600 \\
\text { pixels. }\end{array}$ \\
\hline $\begin{array}{l}\text { Geometric } \\
\text { features based } \\
{[28]}\end{array}$ & $\begin{array}{l}\text { Images captured at } \\
\text { midnight using wide-angle } \\
\text { cameras, such as fisheye- }\end{array}$ \\
\hline
\end{tabular}

The images acquired from a two-month experimental campaign. There were 4 different scenarios with different sensor setups to account for the potential variability between scenarios.

Mean Accuracy = $97.03 \%$

MV classifier gives a recall of $96.8 \%$ and a precision of $98.2 \%$.

Accuracy $=99.67 \%$. lens or catadioptric in the indoor parking area.

\begin{tabular}{|c|c|c|}
\hline $\begin{array}{l}\text { Edge-based + } \\
\text { Custom designed } \\
\text { Deep Learning } \\
\text { model [6] }\end{array}$ & PKLot Dataset & Accuracy $=99.51 \%$ \\
\hline $\begin{array}{l}\text { 3D structural + } \\
\text { Multi-layer } \\
\text { Inference } \\
\text { Framework [22] }\end{array}$ & $\begin{array}{l}\text { They captured a training } \\
\text { image for every } 15 \text { minutes } \\
\text { from 6:30 AM to } 18: 00 \\
\text { PM. For one day, they had } \\
46 \text { training images. Hence, } \\
\text { they had } 92 \text { training } \\
\text { images for } 2 \text { different } \\
\text { weather type. }\end{array}$ & $\begin{array}{l}\text { For dataset DS_5 FPR } \\
=0.0159, \text { FNR } 0.0195, \\
\text { Accuracy }=98.27 \% \\
\text { For dataset DS_6 FPR } \\
=0.0163, \text { FNR } 0.0226, \\
\text { Accuracy }=98.12 \%\end{array}$ \\
\hline
\end{tabular}

3D structural [26]

Figure 2: Accuracy visualization of previous studies.

The accuracy rate of all the pre-existing methods have been illustrated in Figure 3, where the accuracy rate for Geometric Feature Based [29] had given the most accuracy, which is a staggering value of $99.67 \%$. The method based on Edge-based and Deep Learning [6] had given the second-best accuracy of $99.51 \%$. Other methods based on 3D-Structure [26], Convolutional Neural Networks in [5], [10] and [14] also gave very promising results.

\section{B. Review based processing time of various Methods}

Studies of existing research shows that using Convolutional Neural Network-based recognition is implemented to detect or classify a parking space can be done in real-time [14][10]. H. Bura et al. [6] have achieved a minimum time of 7.11 milliseconds to classify the parking space and C. C. Huang et al. [22] have done it 3.61 milliseconds. Some of the paper are briefly compared in table

TABLE 3

EXPERIMENTAL RESULTS OF DIFFERENT METHODS BASED ON PROCESSING TIME

\begin{tabular}{|c|c|c|}
\hline Methods & Training Time & Processing Time \\
\hline $\begin{array}{l}\text { Convolutional } \\
\text { neural network + } \\
\text { Deep Learning- } \\
\text { based [13] }\end{array}$ & $\begin{array}{l}\text { For each single experiment } \\
15 \text { minutes on an NVIDIA } \\
\text { GTX } 980\end{array}$ & $\begin{array}{l}\text { To execute the } \\
\text { classification of } 50 \\
\text { parking spaces per } \\
\text { iteration, it takes }\end{array}$ \\
\hline
\end{tabular}




\begin{tabular}{|c|c|c|}
\hline $\begin{array}{l}\text { Convolutional } \\
\text { neural network- } \\
\text { based [14] }\end{array}$ & Not specified & $\begin{array}{l}\text { around } 15 \text { seconds. } \\
\text { Not specified }\end{array}$ \\
\hline $\begin{array}{l}\text { Convolutional } \\
\text { neural network- } \\
\text { based [10] }\end{array}$ & $\begin{array}{l}\text { The training time is around } \\
\text { six hours }\end{array}$ & $\begin{array}{l}\text { Classification of a } \\
\text { single image can easily } \\
\text { be performed in real- } \\
\text { time. }\end{array}$ \\
\hline $\begin{array}{l}\text { Convolutional } \\
\text { neural network- } \\
\text { based CarNet[5] }\end{array}$ & $\begin{array}{l}\text { The training time for } 500 \\
\text { epochs is around five } \\
\text { hoursGeForce GTX } 1080 \\
\text { Ti graphics card was used. }\end{array}$ & $\begin{array}{l}\text { Classification of a } \\
\text { single image can be } \\
\text { done in real-time. }\end{array}$ \\
\hline $\begin{array}{l}\text { Geometric } \\
\text { features based } \\
{[19]}\end{array}$ & Not specified & $\begin{array}{l}\text { Cannot meet the real- } \\
\text { time requirement. }\end{array}$ \\
\hline $\begin{array}{l}\text { Edge-based + } \\
\text { Custom designed } \\
\text { Deep Learning } \\
\text { model [6] }\end{array}$ & Not specified & $\begin{array}{l}\text { Their designed custom } \\
\text { model takes } 50 \times \\
7.11 \mathrm{~ms}=355.5 \mathrm{~ms} \text { to } \\
\text { classify } 50 \text { slots }\end{array}$ \\
\hline $\begin{array}{l}\text { 3D structural + } \\
\text { Multi-layer } \\
\text { Inference } \\
\text { Framework [22] }\end{array}$ & Not specified & $\begin{array}{l}\text { This experiment takes } \\
\text { an average of } 0.26 \\
\text { seconds to classify } 72 \\
\text { parking spaces. }\end{array}$ \\
\hline
\end{tabular}

Figure 13 shows processing time differences in various researches that were both in real-time and not in real time. The researches that are able to do the processing in real-time [8], [13], [23], [26], [34], [35] while others not done in real time are [19], [30]. So, it is one of the key characteristics to consider while following a method.

\section{OBSERVATION AND DISCUSSION}

This study illustrates different approaches of parking spot vacancy detection based on previous studies and compares them in terms of methods, frameworks and experimental results. Using methods mentioned in Error! Reference source not found.Error! Reference source not found. S. E. Shih et al. [28] were able to achieve accuracy more than $95 \%$ but the methods have limitations in terms of use cases like some can work only in indoor parking environment with limited numbers of vehicle support. There is a lack of suitable methods for automatic parking space extraction, potential difficulties in parking space detection. Previous studies show that, in most of the cases, there was no such method for automatic segmentation of parking spaces, the parking spaces that are used for detection were known while training and testing [13], [23]. In some cases, automatic segmentation of parking space is attempted but it does not have an accuracy that is good enough for real-life scenario, C. Brown et al. [30] mentioned that improvements required to produce better results.

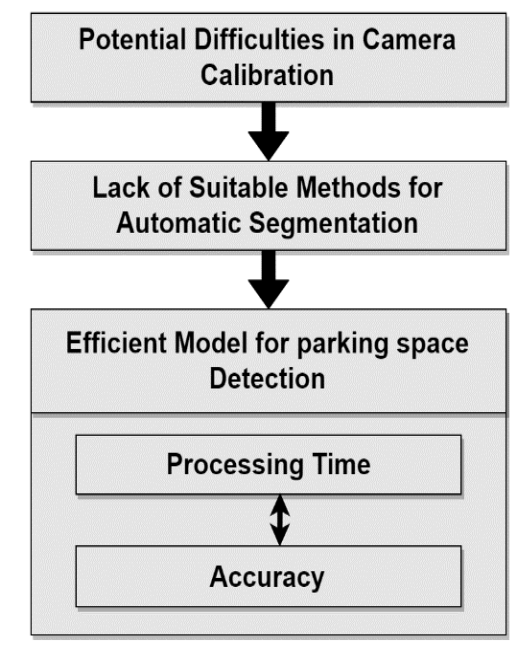

Figure 3: Challenges in Parking space vacancy detection.

Figure 3, shows challenges in parking space vacancy detection. A good method should remove difficulties in camera calibration. It should use security cameras that already exist in the parking area and camera placement and angle change should be allowed on demand for that a suitable method for segmentation of parking spaces is also required. Automatic segmentation may include overhead in processing time but this processing time needs to be minimized for realtime processing while keeping the accuracy high.

\section{CONCLUSION}

This research illustrates the existing methods along with their advantage and disadvantages mentioned in Error! Reference source not found. and also the comparison between experimental results in terms of accuracy on different datasets and processing time is shown. From this study, a number of datasets can be found from different sources that are free to work with and also different methods that are used in various researches to collect parking data. One of the most crucial and important aspects of this research is to address the challenges, in this study different challenges in Parking space vacancy detection have been shown in Figure 3 and also been described in observation and discussion. 


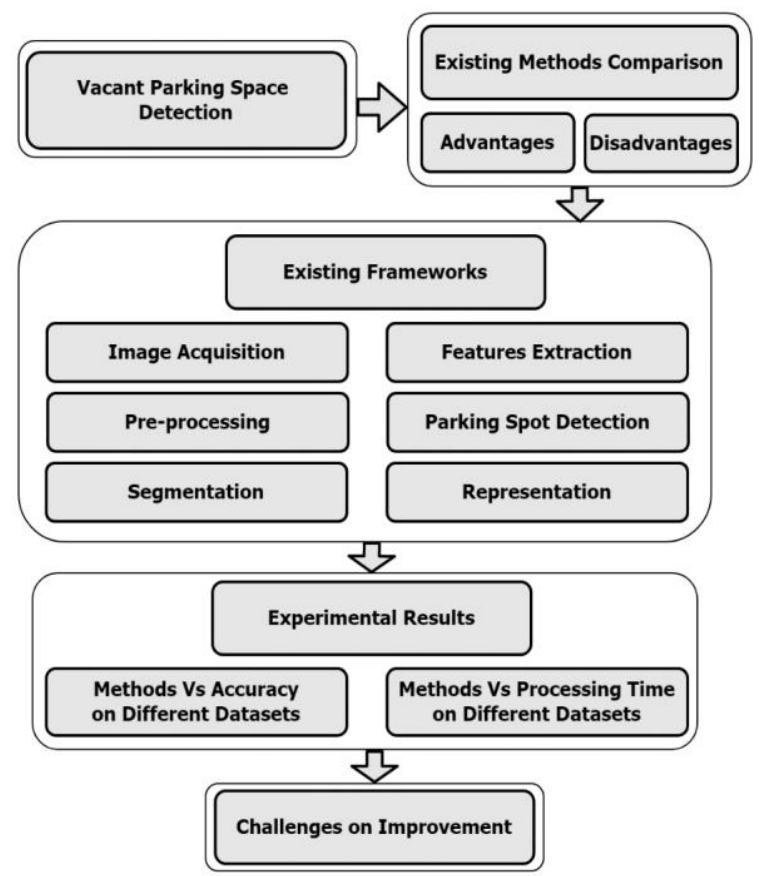

Figure 4: Findings of this Research at a Glance.

Smart parking spot detection is one of the key requirements for smart cities as it has been estimated that "A motorist spends an average of 17 hours per year searching for a place to park his vehicle" [6]. So, the time and work it needs to find a vacant parking space do not worth the effort of a human individual in this era of technology. The complete overview of the research work had been summarized in the Figure 6. This comprehensive review is intended to contribute to Intelligent Transportation System (ITS) research to find aspects that are needed to develop an efficient vacant parking space detection.

\section{REFERENCES}

[1] [1] N. Bibi, M. N. Majid, H. Dawood, and P. Guo, "Automatic Parking Space Detection System," Proc. - 2017 2nd Int. Conf. Multimed. Image Process. ICMIP 2017, vol. 2017-Janua, no. October, pp. 11-15, 2017.

[2] [2] R. M. Nieto, A. Garcia-Martin, A. G. Hauptmann, and J. M. Martinez, "Automatic Vacant Parking Places Management System Using Multicamera Vehicle Detection," IEEE Trans. Intell. Transp. Syst., vol. 20, no. 3, pp. 1069-1080, 2019.

[3] [3] D. Di Mauro, A. Furnari, G. Patanè, S. Battiato, and G. M. Farinella, "Estimating the occupancy status of parking areas by counting cars and non-empty stalls," J. Vis. Commun. Image Represent., vol. 62, pp. 234-244, 2019.

[4] [4] F. Dornaika, K. Hammoudi, M. Melkemi, and T. D. A. Phan, "An efficient pyramid multi-level image descriptor: application to image-based parking lot monitoring," Signal, Image Video Process., 2019.

[5] [5] S. Nurullayev, S. W. Lee, D. Convolutional, and N. Network, "Generalized parking occupancy analysis based on dilated convolutional neural network," Sensors (Switzerland), vol. 19, no. 2, 2019.

[6] [6] H. Bura, N. Lin, N. Kumar, S. Malekar, S. Nagaraj, and K. Liu, "An edge based smart parking solution using camera networks and deep learning," Proc. - 2018 IEEE Int. Conf. Cogn. Comput. ICCC 2018 - Part 2018 IEEE World Congr. Serv., pp. 17-24, 2018.

[7] [7] R. M. Nieto, Á. García-martín, A. G. Hauptmann, and J. M. Martínez, "System Using Multicamera Vehicle Detection," pp. 1-12, 2018.
[8] [8] H. T. Vu and C. C. Huang, "Parking Space Status Inference Upon a Deep CNN and Multi-Task Contrastive Network with Spatial Transform," IEEE Trans. Circuits Syst. Video Technol., vol. 29, no. 4, pp. 1194-1208, 2019.

[9] [9] M. R. Lee and D. T. Lin, "Vehicle counting based on a stereo vision depth maps for parking management," Multimed. Tools Appl., vol. 78, no. 6, pp. 6827-6846, 2019.

[10] [10] J. Martinez Garcia, D. Zoeke, M. Vossiek, J. M. García, S. Member, and D. Zoeke, "MIMO-FMCW radarbased parking monitoring application with a modified convolutional neural network with spatial priors," IEEE Access, vol. 6, pp. 41391-41398, 2018.

[11] [11] I. No and G. N. Sarage, "Study of Various Noise Removal Techniques," vol. 6, no. 1, pp. 174-177, 2015.

[12] [12] C. Jang and M. Sunwoo, "Semantic segmentationbased parking space detection with standalone around view monitoring system," Mach. Vis. Appl., vol. 30, no. 2, pp. 309-319, 2019.

[13] [13] G. Amato, F. Carrara, F. Falchi, C. Gennaro, C. Vairo, and G. Moruzzi, "Car parking occupancy detection using smart camera networks and Deep Learning," Proc. IEEE Symp. Comput. Commun., vol. 2016-Augus, no. Dl, pp. 1212-1217, 2016.

[14] [14] T. H. P. Jensen, H. T. Schmidt, N. D. Bodin, K. Nasrollahi, and T. B. Moeslund, "Parking Space Occupancy Verification - Improving Robustness using a Convolutional Neural Network," no. Visigrapp, pp. 311-318, 2017.

[15] [15] S. Valipour, M. Siam, E. Stroulia, and M. Jagersand, "Parking-stall vacancy indicator system, based on deep convolutional neural networks," 2016 IEEE 3rd World Forum Internet Things, WF-IoT 2016, pp. 655-660, 2017.

[16] [16] L. Baroffio, L. Bondi, M. Cesana, A. E. Redondi, and M. Tagliasacchi, "A visual sensor network for parking lot occupancy detection in Smart Cities," IEEE World Forum Internet Things, WF-IoT 2015 - Proc., pp. 745-750, 2015.

[17] [17] P. R. L. De Almeida, L. S. Oliveira, A. S. Britto, E. J. Silva, and A. L. Koerich, "PKLot-A robust dataset for parking lot classification," Expert Syst. Appl., vol. 42, no. 11, pp. 4937-4949, 2015.

[18] [18] G. Amato, F. Carrara, F. Falchi, C. Gennaro, C. Meghini, and C. Vairo, "Deep learning for decentralized parking lot occupancy detection," Expert Syst. Appl., vol. 72, pp. 327-334, 2017.

[19] [19] Q. Li, C. Lin, and Y. Zhao, "Geometric featuresbased parking slot detection," Sensors (Switzerland), vol. 18 , no. $9,2018$.

[20] [20] Q. Wu, C. Huang, S. Wang, W. Chiu, and T. Chen, "Robust Parking Space Detection Considering Inter-Space Correlation," pp. 659-662, 2007.

[21] [21] J. M. Menéndez, C. G. del Postigo, and J. Torres, "Vacant parking area estimation through background subtraction and transience map analysis," IET Intell. Transp. Syst., vol. 9, no. 9, pp. 835-841, 2015.

[22] [22] C. C. Huang and H. T. Vu, "Vacant Parking Space Detection Based on a Multilayer Inference Framework," IEEE Trans. Circuits Syst. Video Technol., vol. 27, no. 9, pp. 2041-2054, 2017.

[23] [23] M. A. Alimi, I. Masmoudi, A. Jamoussi, and A. Wali, "Trajectory analysis for parking lot vacancy detection system," IET Intell. Transp. Syst., vol. 10, no. 7, pp. 461$468,2016$.

[24] [24] L. Li, C. Li, Q. Zhang, T. Guo, and Z. Miao, "Automatic parking slot detection based on around view monitor (AVM) systems," 2017 9th Int. Conf. Wirel. Commun. Signal Process. WCSP 2017 - Proc., vol. 2017Janua, pp. 1-6, 2017.

[25] [25] M. Ahrnbom, K. Astrom, and M. Nilsson, "Fast Classification of Empty and Occupied Parking Spaces Using Integral Channel Features," IEEE Comput. Soc. Conf. Comput. Vis. Pattern Recognit. Work., pp. 16091615, 2016.

[26] [26] M. Tschentscher, C. Koch, M. König, J. Salmen, and M. Schlipsing, "Scalable real-time parking lot 
classification: An evaluation of image features and supervised learning algorithms," Proc. Int. Jt. Conf. Neural Networks, vol. 2015-Septe, 2015.

[27] [27] L. Zhang, J. Huang, X. Li, and L. Xiong, "VisionBased Parking-Slot Detection: A DCNN-Based Approach and a Large-Scale Benchmark Dataset," IEEE Trans. Image Process., vol. 27, no. 11, pp. 5350-5364, 2018.

[28] [28] S. E. Shih and W. H. Tsai, "A convenient visionbased system for automatic detection of parking spaces in indoor parking lots using wide-angle cameras," IEEE Trans. Veh. Technol., vol. 63, no. 6, pp. 2521-2532, 2014

[29] [29] D. Di Mauro, M. Moltisanti, G. Patane, S. Battiato, and G. M. Farinella, "Park Smart," 2017 14th IEEE Int. Conf. Adv. Video Signal Based Surveillance, AVSS 2017, 2017.

[30] [30] C. Brown, "Using Computer Vision Techniques for Parking Space Detection in Aerial Imagery," Adv. Comput. Vis., pp. 190-204, 2014.

[31] [31] P. Jain and V. Tyagi, "A survey of edge-preserving image denoising methods," 2014.

[32] [32] X. Ling, J. Sheng, O. Baiocchi, X. Liu, and M. E. Tolentino, "Identifying parking spaces \& detecting occupancy using vision-based IoT devices," GIoTS 2017 Glob. Internet Things Summit, Proc., 2017.

[33] [33] C. C. Huang, Y. S. Tai, and S. J. Wang, "Vacan parking space detection based on plane-based bayesian hierarchical framework," IEEE Trans. Circuits Syst. Video Technol., vol. 23, no. 9, pp. 1598-1610, 2013.

[34] [34] X. Xiang, N. Lv, M. Zhai, and A. El Saddik, "RealTime Parking Occupancy Detection for Gas Stations Based on Haar-AdaBoosting and CNN," IEEE Sens. J., vol. 17, no. 19, pp. 6360-6367, 2017.

[35] [35] H. Xie, Q. Wu, B. Chen, Y. Chen, and S. Hong, "Vehicle Detection in Open Parks Using a Convolutional Neural Network," Proc. - 2015 6th Int. Conf. Intell. Syst. Des. Eng. Appl. ISDEA 2015, pp. 927-930, 2016.

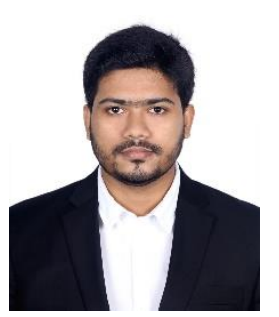

Rifath Mahmud was born on October 15, 1996 in Chattogram, Bangladesh. He received his B.Sc. in Computer Science in 2018 and M.Sc. in Computer Science in 2019 from American International University- Bangladesh (AIUB). Currently, he is working as a Lecturer at the Department of Computer Science at American International University Bangladesh (AIUB). His research interests and passion are mostly based on Computer Vision, Image Processing, Data Analysis, Data Visualization, Artificial Intelligence, and Machine Learning.

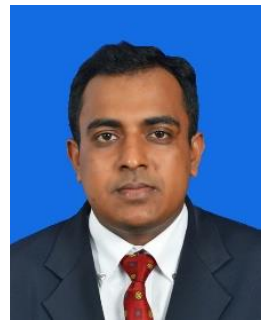

A.F.M. Saifuddin Saif received Ph.D. from Center for Artificial Intelligence and Technology (CAIT), Department of Industrial Computer, Faculty of Information Science and Technology, University Kebangsaan Malaysia (UKM) - The National University of Malaysia in 2016. Dr. Saif served as $\mathrm{PhD}$ research fellow from 2013 till 2015 under various research projects. He received "Outstanding Researcher and Graduate on Time Award (G.O.T.)" from Center for Artificial Intelligence and Technology (CAIT) for his outstanding $\mathrm{PhD}$ research. He received M.Sc. in Computer System Engineering (Software System) from School of Architecture, Computing and Engineering (ACE), University of East London, UK in 2008. Dr. Saif received Best Student Award for his excellent performance during Master study. Dr. Saif received Bachelor of Science (Eng.) degree in Computer Science and Engineering from Shahjalal University of Science and Technology, Bangladesh in 2008, respectively. Most of his contributions in Computer Vision and Artificial Intelligence Research field were published in ISI Q1 journals. He has published many papers in ISI indexed Journals; Scopus indexed Journals, Book Chapters, Conferences, and Proceedings. He served as Technical Committee Members, Reviewers, Guest Speakers, Session Chairs in many Conferences and Workshops. Currently, he is Senior Assistant Professor in department of Computer Science, Faculty of Science and Technology, American International University Bangladesh (AIUB). Before joining the university, he did Post Doctorate at Faculty of Information Science and Technology, University Kebangsaan Malaysia. He spent more than 6 years in IT industry such as Advanced Software Development, Web eMaze etc as IT researcher. His research interests include Image Processing, Computer Vision, Artificial Intelligence, Augmented Reality, 3D Reconstruction, and Medical Image Processing.

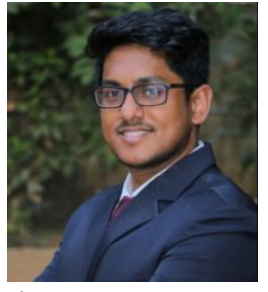

Dipta Gomes was born on March 29, 1994 in Dhaka, Bangladesh. He has completed his B.Sc. in Computer Science in 2018 and M.Sc. in Computer Science in 2019 from American International University- Bangladesh (AIUB). Currently, he is working as a Lecturer at the Department of Computer Science at American International University Bangladesh (AIUB). His research interest includes Graph Theory Algorithms, Computer Vision, Machine Learning, and Image Processing. 